\title{
Erratum: Many-body localization and thermalization in disordered Hubbard chains [Phys. Rev. A 92, 041601(R) (2015)]
}

\author{
Rubem Mondaini and Marcos Rigol \\ (Received 28 October 2015; published 6 November 2015)
}

DOI: 10.1103/PhysRevA.92.059902

PACS number(s): 67.85.-d, 05.30.-d, 71.30.+h, 99.10.Cd

The normalization of the results for the Aubry-André model reported in the Fig. 1(e) was incorrect. The corrected figure is enclosed. This does not affect any of the conclusions in the paper, except that $\Delta_{c}<W_{c}$ and not much smaller as originally stated.

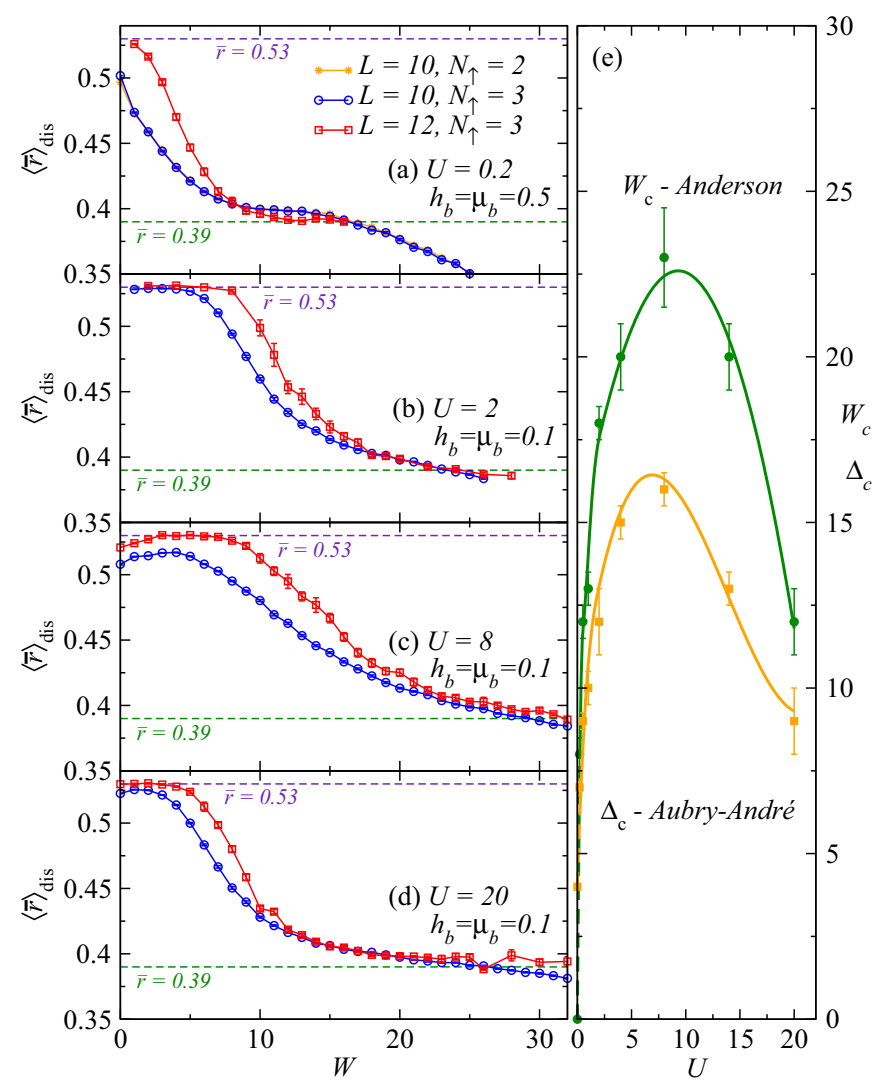

FIG. 1. (Color online) (a)-(d) Averaged ratio of adjacent energy gaps as a function of the disorder strength for four values of $U$ and two lattice sizes. The average $\bar{r}$ was computed over the central half of the spectrum. The disordered averaged results $\langle\bar{r}\rangle_{\text {dis }}$ for $L=10$ were obtained by averaging over 1200 disorder realizations and the ones for $L=12$ over 20-200 disorder realizations (error bars report the standard deviation). In (a) we show results for $N_{\uparrow}=N_{\downarrow} \pm 1$ when $L=10$. They make apparent that both sectors behave qualitative (and quantitatively) similarly even for the largest values of $h_{b}=\mu_{b}$ used. The crossing point between curves for different lattice sizes provides an estimate of the critical disorder $W_{c}$ for the ergodic to MBL transition. (e) Estimated $W_{c}$ and $\Delta_{c}$ as a function of $U$ (error bars report an interval of confidence based on the closeness of the results about $W_{c}$ ). 